\title{
LATTICES OF FUZZY OBJECTS
}

ARTURO A.L. SANGALLI

\section{Department of Mathematics, Champlain Regional College}

P.O. Box 5003, Lennoxville, Quebec, Canada J1M 2 A1

(Received October 27, 1994 and in revised form March 8, 1995)

\begin{abstract}
The collection of fuzzy subsets of a set $X$ forms a complete lattice that extends the complete lattice $\boldsymbol{P}(X)$ of crisp subsets of $X$. In this paper, we interpret this extension as a special case of the "fuzzification" of an arbitrary complete lattice $A$. We show how to construct a complete lattice $F(A, L)$--the Lfuzzification of $A$, where $L$ is the valuation lattice-- that extends $A$ while preserving all suprema and infima. The "fuzzy" objects in $F(A, L)$ may be interpreted as the sup-preserving maps from $A$ to the dual of $L$. In particular, each complete lattice coincides with its 2 -fuzzification, where 2 is the twoelement lattice. Some familiar fuzzifications (fuzzy subgroups, fuzzy subalgebras, fuzzy topologies, etc.) are special cases of our construction. Finally, we show that the binary relations on a set $X$ may be seen as the fuzzy subsets of $X$ with respect to the valuation lattice $\mathbf{P}(X)$.
\end{abstract}

KEY WORDS. Complete lattices, fuzzification, fuzzy subalgebras.

AMS SUBJECT CLASSIFICATION. Primary 03E72; secondary 06B99.

\section{INTRODUCTION.}

The theory of fuzzy sets began with Zadeh's definition [1] of a fuzzy subset of a set $X$ as a map from $X$ to the unit interval $I$ ( $=$ the set of real numbers from 0 to 1$)$. Since $I$ is a complete lattice for the natural order $\leq$, the collection of all fuzzy subsets of $X$ is also a complete lattice with respect to the order defined pointwise, i.e. $f \leq g$ if $f(x) \leq g(x)$ for all $x \in X$. We denote by $P_{I}(X)$ the lattice of all fuzzy subsets of $X$.

The set of (crisp) subsets of $\mathrm{X}$ ordered by inclusion also forms a complete lattice $P(X)$ whose elements may be identified in the usual way with the maps from $X$ to $I$ whose values are either 0 or 1 --the characteristic functions of subsets of $X$. Under this identification, the lattice $P_{Y}(X)$ is an extension of $P(X)$ that preserves all suprema and infima. In this sense, $P_{I}(X)$ may be considered as a kind of "fuzzification" of $\mathbf{P}(X)$.

In this paper we show how going from $P(X)$ to $P_{I}(X)$ may be generalised in two directions. Firstly, $\mathbf{P}(X)$ may be replaced by an arbitrary complete lattice $A$, such as the lattice of subgroups of a given group, the ideals of a ring or the closed sets in a topological space. We then construct a complete lattice $F(A, I)--$ the "fuzzification" of the given complete lattice A-- that plays the role of $P_{I}(X)$. This fuzzification is such that there is a lattice embedding $A-->F(A, I)$ 
preserving all suprema and infima. Moreover, the usual definitions of fuzzy subsets, fuzzy subgroups (Rosenfeld [2]), fuzzy topology (Lowen [3]), etc. are special cases of our construction. Thus, we obtain a uniform procedure for fuzzifying concepts such as "subalgebra". "closed set" and others having the property that the crisp objects form a complete lattice.

The second generalisation consists in replacing I by an arbitrary complete lattice $L$ called the valuation lattice. For each complete lattice $A$ we then obtain its L-fuzzification $F(A, L)$. This is a complete lattice that extends $A$ while preserving all suprema and infima. As an example, we show that the $P(X)$ fuzzification of $\boldsymbol{P}(X)$, i.e. $F(P(X), P(X))$, is isomorphic to the dual of the lattice of binary relations on the set $X$. Thus, the binary relations on a set $X$ may be seen as the fuzzy subsets of $X$ with respect to the valuation lattice $P(X)$.

If $L=2$ (= the two-element lattice), the embedding $A-->F(A, 2)$ is an isomorphism, so that each complete lattice "is" its own 2-fuzzification.

\section{CLOSURE SPACES AND RESIDUATED MAPS}

In order to proceed with the generalisations, we must look at $\boldsymbol{P}_{\mathbf{I}}(\mathrm{X})$ in a different way, not merely as the set of all maps from $X$ to $I$. Both $X$ and $I$ are in fact what we call below "closure spaces", and the elements of $P_{\mathrm{T}}(\mathrm{X})$ are precisely the "continuous" maps from $X$ to $I$. Also, these maps are in one-to-one correspondence with the "residuated" maps (defined below) from the complete lattice $\boldsymbol{P}(X)$ to the complete lattice $I$. Thus, we can describe the fuzzification of $\mathbf{P}(X)$ in terms of either continuous maps between closure spaces or residuated maps between complete lattices.

Definition 2.1. A closure space (Achache and Sangalli [4]) is a pair ( $\mathrm{S}, \mathfrak{F})$, where $S$ is a set and $F$ is a collection of subsets of $S$, called closed subsets, with the property that the intersection of any family of closed subsets is a closed subset.

In particular $S$ (= intersection of the empty family) is closed. Thus, $F$ is a complete lattice with respect to inclusion of subsets, where infima coincide with intersections but suprema are not in general equal to unions.

Examples of closure spaces are:

(1) $(X, P(X))$, for any set $X$.

(2) $S$ is the base set of a group $G$ and $F=$ the collection of all subgroups of $G$.

(3) $S$ is the base set of an universal algebra $\mathcal{A}$ and $F=$ the subalgebras of $\mathcal{A}$.

(4) $(T, F)$, where $(T, U)$ is a topological space and $F$ is the collection of all sets closed in the topology $U$.

If $(S, F)$ is a closure space, then each subset $X$ of $S$ has a closure $X^{-}$, defined as the smallest closed set containing $X$ (e.g., in example (2) above, $X^{-}=$the subgroup generated by $X)$. The closure of the singleton $\{x\}$ will be denoted $x^{-}$.

Definition 2.2. A continuous map between two closure spaces $\left(\mathrm{S}_{1}, \boldsymbol{F}_{1}\right)$ and $\left(S_{2}, F_{2}\right)$ is a map $f: S_{1} \quad \ldots S_{2}$ such that the inverse image $f^{-1}(X)$ of each closed subset $X$ is closed. The set of all such maps will be denoted $\operatorname{Cont}\left(\left(S_{1}, F_{1}\right),\left(S_{2}, F_{2}\right)\right)$.

If $(P, \leq)$ is a partially ordered set and $p \in P$, then $(p]=\{x \in P: x \leq p\}$ is the principal ideal generated by $\mathrm{p}$ and $[\mathrm{p})=\{\mathrm{x} \in \mathrm{P}: \mathrm{p} \leq \mathrm{x}\}$ the principal filter generated by $\mathrm{p}$. 
Definition 2.3. A map $f: P \ldots$ : $P$ between ordered sets is called residuated if for all $q \in Q$ there exists $p \in P$ such that $f^{-1}(q]=(p] \cdot \operatorname{Res}(P . Q)$ denotes the set of residuated maps from $P$ to $Q$.

If $P$ and $Q$ are complete lattices, then the following are properties of residuated maps (see [5] for the proofs).

(a) $f: P \ldots P$ is residuated iff $f$ is isotone (i.e. order-preserving) and there exists an isotone map $f \#: Q-->P$ satisfying: for all $p \in P$, for all $q \in Q, f(p) \leq q \Leftrightarrow$ $p \leq f \#(q)$. This map $f \#$ is called the residual of $f$ and $f \#(q)$ is the unique $p \in P$ such that $f^{-1}(q]=(p]$. If $P^{*}$ denotes the dual of $P$, i.e. $P^{*}=(P, \geq)$, then $f \# \in \operatorname{Res}\left(Q^{*}\right.$. P*).

(b) $f: P$---> $Q$ is residuated iff $f$ preserves all suprema.

(c) $\operatorname{Res}(P, Q)$ is a complete lattice, with $f \leq g$ iff $f(x) \leq g(x)$ for all $x \in P$, and $\left(\sup f_{j}\right)(x)=\sup \left(f_{j}(x)\right)$, for each family $\left\{f_{j}: j \in J\right\}$ of residuated maps.

\section{THE ADJUNCTION BETWEEN F AND $\underline{L}$}

The precise relationship between closure spaces and residuated maps is best expressed in the language of category theory using the notion of an adjunction. We shall do so in the sequel and refer the reader to [6] for the relevant definitions (of category, functor, adjunction, etc.) and results. Nevertheless, the reader not familiar with --or not interested in-- categories may still get the gist of it from Theorem 3.1.

Since identity maps and compositions of residuated [respectively, continuous] maps are residuated [continuous], there is a category $\underline{L}$ whose objects are complete lattices and whose morphisms are residuated maps and a category $\underline{F}$ with closure spaces as objects and continuous maps as morphisms.

Every complete lattice $L$ becomes a closure space $(L, P(L))$ by taking as its closed sets the collection $P(L)$ of all principal ideals of $L$. This correspondence defines a functor $D$ : $\underline{L}-->\underline{F}$. Since every morphism in $\underline{L}$ from $L_{1}$ to $L_{2}$ is also a morphism in $\underline{F}$ from $D\left(L_{1}\right)$ to $D\left(L_{2}\right)$ and conversely, the functor $D$ embedds $\underline{L}$ as a full subcategory of $\underline{F}$. The following characterisation of $D(\underline{L})$ is proved in [4] (Proposition 4): " $D(\underline{L})$ is the full subcategory of $\underline{F}$ whose objects are the closure spaces with the property that each closed subset $X$ is of the form $x^{-}$for a unique $\mathrm{x} \in \mathrm{X}$ ".

The existence of a left adjoint to $D$ follows from:

Lemma 3.1. Let $(S, F)$ be a closure space and $L$ a complete lattice. Let $\eta_{S}$ : $S-->F$ be the map that associates to $x \in S$ its closure, i.e. $\eta_{S}(x)=x^{-}$. Then, for each continuous map $v:(S, F)-\ldots(L, P(L))$, there exists exactly one residuated map $v^{\prime}: F-\rightarrow L$ such that $v^{\prime} \circ \eta_{S}=v$.

Proof. The map $v^{\prime}$ is defined by $v^{\prime}(X)=\sup v(X)$, for all $X \in F$. The verification that $v^{\prime}$ preserves suprema makes use of the following characterisation of the set $\operatorname{Cont}((S, F),(L, P(L)))([4]$, Proposition 3):

"A map v: $S-\rightarrow L$ is continuous iff for all $X \subseteq S$, $\sup v(X)=\sup v\left(X^{-}\right) "$. Let $\left\{X_{k}: k \in K\right\} \subseteq F$. Then $v^{\prime}\left(\sup _{k \in K} X_{k}\right)=v^{\prime}\left(\left(U_{k} \in K X_{k}\right)^{-}\right)=\sup v\left(\left(U_{k} \in K X_{k}\right)^{-}\right)=\sup$ $v\left(U_{k} \in K X_{k}\right)=\sup \left\{v(a): a \in U_{k} \in K X_{k}\right\}=\sup _{k} \in K \sup v\left(X_{k}\right)=\sup _{k \in K} v^{\prime}\left(X_{k}\right)$.

Now let $x \in S$. Then $x \in v^{-1}((v(x)])$. Since $v$ is continuous, $v^{-1}((v(x)]) \in F$. By definition of closure, we then have $x^{-} \subseteq v^{-1}((v(x)])$. This means that, for all $y \in$ 
$x^{-}, v(y) \leq v(x)$. Hence $v(x) \leq \sup \left\{y: y \in x^{-}\right\} \leq v(x)$ and therefore sup $v\left(x^{-}\right)=v(x)$. i.e. $v^{\prime} \circ \eta_{S}=v$.

To check uniqueness, suppose that $h: F \rightarrow L$ preserves suprema and satisfies $h \circ \eta_{S}=v$. Let $X \in F$ (this implies $X=\sup \left\{x^{-}: x \in X\right\}$ ). Then we have $h(X)$ $=h\left(\sup \left\{x^{-}: x \in X\right\}\right)=\sup \left\{h\left(\eta_{S}(x)\right): x \in X\right\}=\sup \{v(x): x \in X\}=\sup v(X)$. Hence $h$ $=v^{\prime} . \diamond$ (The symbol $\diamond$ indicates the end of a proof $)$.

Remark (for readers familiar with category theory): the map $\eta_{S}$ defined above is a continuous map from $(S, \mathcal{F})$ to the closure space $(F, P(F))(=D(\mathcal{F})$ ). Then. Lemma 3.1 merely states that the pair $\left(S, \eta_{S}\right)$ is a universal morphism from $(S, F)$ to $D$. It is well-known that the existence of such a morphism for each object of $\underline{F}$ implies the existence of a left adjoint $C: \underline{F}-->\underline{L}$ to the functor $D(C$ is defined on objects by $\mathrm{C}((\mathrm{S}, \mathcal{F}))=\mathcal{F}$. For a morphism $h:\left(S_{1}, F_{1}\right) \cdots\left(S_{2}, F_{2}\right)$ of $\underline{F}$, $C(h): F_{1} \ldots F_{2}$ is the closure of the image, i.e. for all $\left.X \in F_{1}, C(h)(X)=h(X)^{-}\right)$. The map $\beta$ in the next theorem arises when this adjunction is reformulated in terms of hom sets.

Theorem 3.1. The map $\beta$ : $\operatorname{Cont}((S, F),(L, P(L)))-->\operatorname{Res}(F, L)$, defined by $\beta(v)(X)=\sup v(X)--$ for all continuous $v: S-->L$ and all $X \in F$ - is a bijection whose inverse $\alpha$ is given by $\alpha(r)(x)=r(\{x\})$, where $r \in \operatorname{Res}(F, L)$ and $x \in S$. Moreover, since both $\alpha$ and $\beta$ preserve order, they are isomorphisms of complete lattices.

Proof. It follows from Lemma 3.1 that $\beta(v) \in \operatorname{Res}(F, L)$, and it is easy to check that $\beta$ is a bijection with inverse $\alpha$. Cont $((S, F),(L, P(L)))$ is a subset of the complete lattice of all maps from $S$ to $L$. To prove that it is itself a complete lattice, it is sufficient to show that the supremum (computed pointwise) of a family $\left\{f_{j}: j \in J\right\}$ of continuous maps from $S$ to $L$ is continuous. It is easy to verify the equation

$\left(\sup _{j \in J} f_{j}\right)^{-1}((a])=\bigcap_{j \in J} \quad f_{j}^{-1}((a])$, for all $a \in A$.

Now, each $f_{j}^{-1}((a])$ is a closed subset of $S$, for $f_{j}$ is continuous; hence, the right-hand side of equation (3.1) is also a closed subset of $S$, being the intersection of a family of closed subsets. Finally, it is routine to check that both $\alpha$ and $\beta$ preserve order. $\diamond$

\section{THE FUZZIFICATION}

Recall that $L^{*}$ denotes the dual of the lattice $L$. Let $A$ be an arbitrary complete lattice and let 2 denote the two-element lattice. For each a $\in A$, the map $f_{a}: A-\rightarrow>2$ defined by $f_{a}(x)=0$ iff $x \leq a$ is residuated, so each element of $A$ may be seen as a residuated map from $A$ to 2 . Conversely, each residuated map from $A$ to 2 is determined in this way by some a $\in A$. Thus, the correspondence $f_{(-)}: A-\rightarrow \operatorname{Res}(A, 2)$ is a bijection that reverses order and whose inverse is also order-reversing. Therefore, we have shown:

Lemma 4.1. $f_{(-)}$is a lattice isomorphism from $A$ to $\operatorname{Res}(A, 2) *$.

Let $L$ be a complete lattice. Notice that both $\operatorname{Res}(A, 2)$ and $\operatorname{Res}(A, L)$ are complete lattices (Property (c), section 2). The map $\Phi: \operatorname{Res}(A, 2)-->\operatorname{Res}(A, L)$ is defined as follows: if $f$ is a residuated map from $A$ to 2 , then $\Phi(f)$ is $f$ viewed as a map from $A$ to $L$ (more precisely, $\Phi(f)$ is the composition of $f$ with the unique 
lattice homomorphism $2 \ldots \mathrm{L}$ that preserves 0 and 1). It is shown in [7] that $\Phi$ is a lattice embedding that preserves all suprema and infima. Hence, $\Phi: \operatorname{Res}(A$, $2)^{*} \ldots \rightarrow \operatorname{Res}\left(\mathrm{A}, \mathrm{L}^{*}\right)^{*}$ also preserves all suprema and infima. Now, by Lemma 4.1 , $A$ is isomorphic to $\operatorname{Res}(A, 2)^{*}$. Thus, $\operatorname{Res}\left(A, L^{*}\right)^{*}$ is an extension of $A$ that preserves all suprema and infima. We call it the L-fuzzification of A.

Definition 4.1. Let $L$ be a complete lattice called the valuation lattice and let $A$ be a given complete lattice. The $L$-fuzzification of $A$, denoted $F(A, L)$, is the complete lattice $\operatorname{Res}\left(A, L^{*}\right) *$.

We have proved above:

Theorem 4.1. The L-fuzzification of a complete lattice $A$ is an extension of A that preserves all suprema and infima.

Theorem 4.2. Every complete lattice $A$ is isomorphic to its 2 -fuzzification.

Proof. Let $\approx$ indicate lattice isomorphism. Then, since $2 \approx 2^{*}$, we have $F(A$, 2) $=\operatorname{Res}\left(\mathrm{A}, 2^{*}\right)^{*} \approx \operatorname{Res}(\mathrm{A}, 2)^{*} \approx \mathrm{A}$, where the last isomorphism follows from Lemma 4.1 .0

\section{APPLICATIONS}

In this section we show that many lattices of fuzzy objects (fuzzy subsets, fuzzy closed sets, fuzzy subalgebras, etc.) arise as special cases of our construction., i.e. they are (isomorphic to) the fuzzification of the complete lattice of crisp objects (subsets, closed sets, subalgebras, etc.).

\section{FIZZY SL BSETS}

Theorem 5.1. The complete lattice $P_{Y}(X)$ of fuzzy subsets of a set $X$ is isomorphic to the I-fuzzification of $\mathbf{P}(\mathrm{X})$.

Proof. By Theorem 3.1, we have that $\operatorname{Res}\left(\mathcal{P}(X), I^{*}\right)$ is isomorphic to $\operatorname{Cont}((X$, $\mathbf{P}(X))$, (I*, $\left.\left.\mathrm{P}\left(\mathrm{I}^{*}\right)\right)\right)$. Now, this latter lattice is identical with $\operatorname{Cont}((X, \mathbf{P}(X)),(\mathrm{I}, \mathrm{F}(\mathrm{I})))^{*}$, where $F(I)$ denotes the family of principal filters of I. By taking the dual on both sides we get that

$$
\operatorname{Res}\left(\boldsymbol{P}(\mathrm{X}), \mathrm{I}^{*}\right)^{*} \approx \operatorname{Cont}((\mathrm{X}, \mathbf{P}(\mathrm{X})),(\mathrm{I}, \mathrm{F}(\mathrm{I})))
$$

Finally, the left-hand side of $(5.1)$ is by definition $F(P(X), I)$ while the righthand side is the set of all functions from $X$ to $I\left(=P_{I}(X)\right) . \diamond$

\section{FUZZY SLBGROUPOIDS}

Let $U$ be a groupoid, i.e. a set equipped with a binary operation. A fuzzy subgroupoid (Novak [8]) of $U$ is a map f: $U$-.-> $L$, where $L$ is the valuation lattice, such that for all $x, y \in U$,

$$
f(x) \wedge f(y) \leq f(x y)
$$

Let $S(U)$ denote the complete lattice of (crisp) subgroupoids of $U$ and $F(L)$ the collection of principal filters of $L$. As in (5.1) above, it is easy to see that Cont((U, S(U)), (L, $F(L)))$ is isomorphic to the L-fuzzification $F(S(U), L)$ of $S(U)$.

Theorem 5.2. $f: U$---> $L$ is a fuzzy subgroupoid of $U$ if and only if $f \in$ Cont((U, S(U)), (L, F(L))).

Proof. To show that $f$ is a continuous map, it suffices to check that $f^{-1}[q)$ is a subgroupoid of $U$ for all $q \in L$. Let $x, y \in f^{-1}[q)$, i.e. $q \leq f(x)$ and $q \leq f(y)$. Then $q$ $\leq f(x) \wedge f(y)$. From this follows $q \leq f(x y)$ (by transitivity, from (5.2)). Hence $x y \epsilon$ $\mathrm{f}^{-1}[\mathrm{q})$. 
To prove the converse, notice that $f(x) \wedge f(y) \leq f(x)$ and $f(x) \wedge f(y) \leq f(y)$. This implies $x, y \in f^{-1}\left[f(x) \wedge f(y)\right.$ ). Since $f^{-1}[f(x) \wedge f(y)$ ) is a subgroupoid (for $f$ is continuous), we get $x y \in f^{-1}[f(x) \wedge f(y))$, i.e. $f(x) \wedge f(y) \leq f(x y) . \diamond$

\section{FI TIY SI BALGEBRAS}

Let $\mathbf{A}=\langle A, \Omega\rangle$ be an (universal) algebra, i.e. $\Omega$ is a set of finitary operations on the set $A$. Each $n$-ary operation $f$ in $\Omega$ induces an $n$-ary operation $f$ on the set IA of all functions from $A$ to $I$, as follows:

$f\left(m_{1}, \ldots, m_{n}\right)(x)=\sup _{y \in f}-1(\{x\}) \min _{j}\left\{m_{j}\left(y_{j}\right)\right\}$, where $y=\left(y_{1}, \ldots, y_{n}\right)$. (This definition generalises the operation that assigns to the (crisp) subsets $A_{1}, \ldots, A_{n}$ of $A$, the subset $\left.\left\{f\left(a_{1}, \ldots, a_{n}\right): a_{j} \in A_{j}, j=1, \ldots, n\right\}\right)$.

A fuzzy subset $m \in I^{A}$ is said to be a fuzzy subalgebra of $A$ (Murali [9]) if $f(m, m, \ldots, m) \leq m$, for all $f \in \Omega$.

The fuzzy subalgebras of an algebra $A$ form a complete lattice. It is shown in [10] that this lattice is a continuous lattice. For the definition and a thorough study of continuous lattices see [11], where it is shown that a lattice $L$ is continuous iff it is isomorphic to a subset of $\mathrm{I}^{\mathrm{X}}$ which is closed under arbitrary infima and suprema of directed families.

Theorem 5.3. The lattice of fuzzy subalgebras of an algebra $A$ is isomorphic to the I-fuzzification of the complete lattice $S(A)$ of (crisp) subalgebras of $\mathbf{A}$.

Proof. The proof makes use of Theorem 3.1, according to which

$\operatorname{Res}\left(\mathrm{S}(\mathrm{A}), \mathrm{I}^{*}\right) \approx \operatorname{Cont}\left((\mathrm{A}, \mathrm{S}(\mathrm{A})),\left(\mathrm{I}^{*}, \mathrm{P}\left(\mathrm{I}^{*}\right)\right)\right)$.

Now, the right-hand side of $(5.3)$ is identical with the lattice $\operatorname{Cont}((A, S(A))$, (I, $F(I)))^{*}$, where $F(I)$ denotes the collection of principal filters of $I$. By taking the dual on both sides of $(5.3)$ we get $\operatorname{Res}\left(S(A), I^{*}\right)^{*} \approx \operatorname{Cont}((A, S(A)),(I, F(I)))$. This means that the I-fuzzification of $S(A)$--i.e. $\operatorname{Res}\left(S(A), I^{*}\right)^{*--}$ is isomorphic to the lattice of continuous functions from $(A, S(A))$ to $(I, F(I))$. Thus, in order to prove the theorem, it is enough to check that $m$ : $A$---> I is a fuzzy subalgebra of $A$ iff it is a continuous function from $(A, S(A))$ to $(I, F(I))$, i.e. iff for every real number $r$ in $I$, the set $\{a \in A: r \leq m(a)\}$ is closed under all the operations in $\Omega$. A proof of this appeared in [7] (Theorem 5.1). 0

\section{FUZZY TOPOLOGIES}

Let $T$ be a topological space and let $F$ be the complete lattice of closed (in the topology) subsets of $T$. Then $(T, F)$ is a closure space. If $L$ is the valuation lattice we have, by Theorem 3.1, the lattice isomorphism Cont ((T, F), (L, P(L))) $\approx$ $\operatorname{Res}(F, L)$. As in the previous examples, it follows from this that

$\operatorname{Res}\left(F, L^{*}\right)^{*} \approx \operatorname{Cont}((T, F),(L, F(L)))$

Now, let $U$ be the smallest topology on $L$ such that every principal filter (of the lattice $L$ ) is closed in $U$. Then the right-hand side of (5.4) is exactly the set of continuous (in the topological sense) functions from $T$ to $L$ endowed with the above topology. Hence, the fuzzification of a given topology $\tau$ is nothing else than the fuzzy topology $\omega(\tau)$ introduced in [3]. 


\section{RELATIONS SEEN AS FUZZY SUBSETS}

Given a set $X$, let $R(X)$ be the set of all binary relations on $X$, i.e. subsets of $X \times X$. Then $R(X)$ is a complete lattice under inclusion. We now show that the $\boldsymbol{P}(X)$-fuzzification of $\mathbf{P}(X)$ is isomorphic to the dual of the lattice $\boldsymbol{R}(X)$. Thus, a binary relation on a set $X$ may be seen as a $P(X)$-fuzzy subset of $X$. Morcover. this one-to-one correspondence is such that the lattice structure is (dually) preserved.

Let $\mathrm{YX}$ denote the collection of all maps from the set $\mathrm{X}$ to the set $\mathrm{Y}$. There is a bijection $r$ : $P(X) X \ldots P(X)$ which sends $f: X \ldots P(X)$ to the binary relation $r(f)$ on $X$ defined by $x r(f) y \Leftrightarrow y \in f(x)$, for all $x, y \in X$. The inverse bijection associates to the relation $\rho$ on $X$, the map $X-->\mathcal{P}(X)$ that sends $x$ to $\{y \in X: x \rho y\}$.

There is also a bijection s: $\boldsymbol{P}(X)^{\mathcal{P}(X) \ldots P} \boldsymbol{P}(X) X$ which associates to $f$ its "restriction to singletons" --more precisely, $s(f)(x)=f(\{x\})$. (The bijection $s$ is "natural" in the sense of category theory since it arises from an adjunction: the power-set functor $P$ : $\underline{S}$---> $\underline{L}$, where $\underline{S}$ is the category of sets, is left adjoint to the forgetful functor from complete lattices to sets --i.e. $P(X)$ is the "free" object generated by $X$ in the category $\underline{L}$ ).

The composition of the above bijections produces the bijection $R: \operatorname{Res}(P(X)$, $\boldsymbol{P}(X)) \ldots \boldsymbol{R}(X)$ that associates to a given residuated map $f: P(X) \ldots>P(X)$, the relation $R(f)$ on $X$ defined by: $x R(f)$ y $\Rightarrow y \in f(\{x\})$. It is straightforward to verify that the bijection $R$ preserves and reflects order and, therefore, that it is an isomorphism of lattices.

The $\boldsymbol{P}(X)$-fuzzification of $\boldsymbol{P}(X)$ is, by definition, the complete lattice $\operatorname{Res}\left(\boldsymbol{P}(X), \boldsymbol{P}(X)^{*}\right)^{*}$. Since $\boldsymbol{P}(X)^{*}$ is isomorphic to $\boldsymbol{P}(X)$, we have proved:

Theorem 6.1. For every set $X$, the $\boldsymbol{P}(X)$-fuzzification of $\boldsymbol{P}(X)$ is dually isomorphic to the complete lattice of binary relations on $\mathrm{X}$.

A quantale [12] is a complete lattice $\mathrm{Q}$ together with an associative product (denoted " $\&$ ") which distributes over arbitrary suprema. For example, the lattice $\boldsymbol{R}(\mathrm{X})$ is a quantale with respect to the composition of relations. This structure on $\boldsymbol{R}(\mathrm{X})$ has been applied implicitly by Hoare and He [13] in the case where $X$ is a set of states of a machine to construct the weakest prespecification of a program with respect to another.

From an argument similar to the above it follows that the quantale $Q(H)$ of linear relations on a Hilbert space $H$ may be seen as the $\mathrm{C}(\mathrm{H})$-fuzzification of the complete lattice $\mathbf{C}(\mathbf{H})$ of all closed linear subspaces of $\mathbf{H}$.

ACKNOWLEDGEMENTS. The author was supported by a grant from Quebec's Fund FCAR.

\section{REFERENCES}

1. ZADEH, L.A. Fuzzy sets, Information and Control 8 (1965), 338-353.

2. ROSENFELD, A. Fuzzy groups, J. Math. Anal. Appl. 35 (1971), 512-517.

3. LOWEN, R. Fuzzy topological spaces and fuzzy compactness. J. Math. Anal. Appl. 56 (1976), 621-633. 
4. ACHACHE, A. and SANGAlli, A.A.L. Préordres, résiduation et espaces de fermeture, Discrete Math. 130 (1994), 3-7.

5. CROWN, G.D. Projectives and Injectives in the Category of Complete Lattices with Residuated Mappings, Math. Ann. 187 (1970), 295-299.

6. BARR, M. and WELLS, C. Category Theory for Computing Science, Prentice Hall, 1990.

7. SANGALLI, A.A.L. Fuzzifications of complete lattices and applications, Proc. of the 2nd International Conference on Fuzzy Logic and Neural Networks. lizuka (1992), 597-600.

8. NOVAK, V. Fuzzy Sets and their Applications, Adam Hilger, Bristol and Philadelphia, 1989.

9. MURALI, V. Lattice of fuzzy subalgebras and continuous lattices, Proceedings of IFSA '9I, Mathematics Vol., Brussels (1991), 141-144.

10. MURALI, V. On lattice of fuzzy subalgebras and closure systems in IX", Fuzzy Sets and Systems 41 (1991), 101-111.

11. GIERZ, G., HOFMAN, K.H. et al. A compendium of continuous lattices, SpringerVerlag, Berlin, Heidelberg, New York, 1980.

12. MUlVeY, C.J. \&, Rendiconti Circ. Mat. Palermo 12 (1986), 99-104.

13. HOARE, C.A.R. and HE, J. The weakest prespecification, Information Processing Letters 24 (1987), 127-132. 


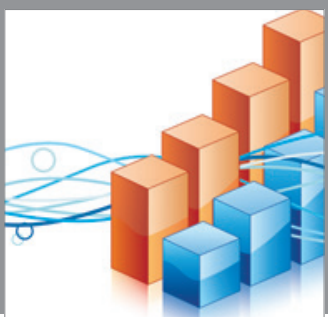

Advances in

Operations Research

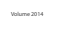

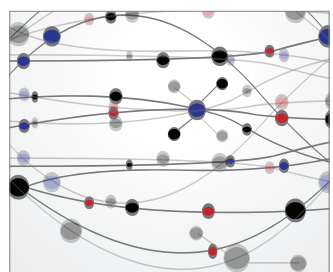

\section{The Scientific} World Journal
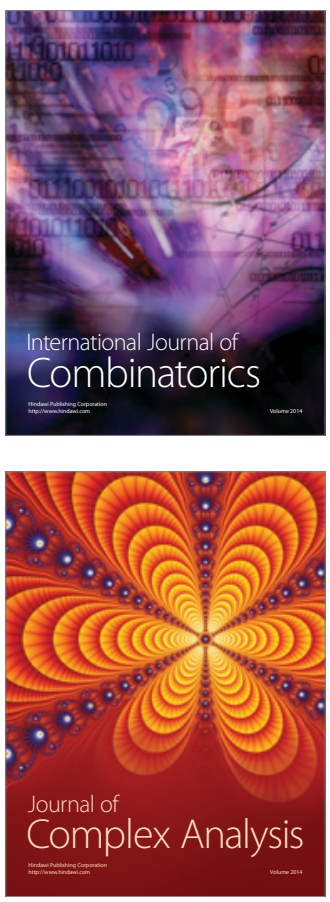

International Journal of

Mathematics and

Mathematical

Sciences
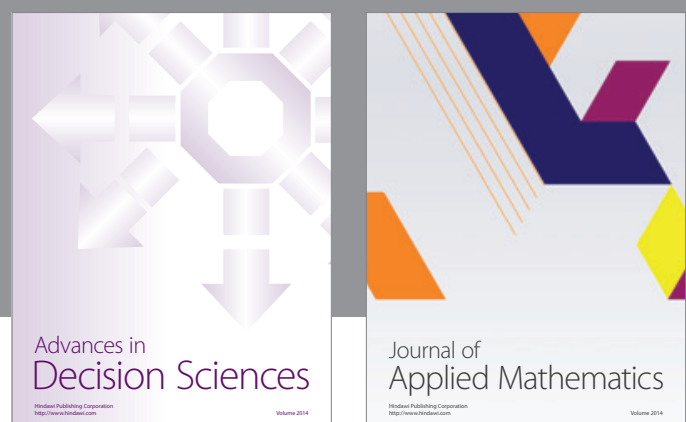

Journal of

Applied Mathematics
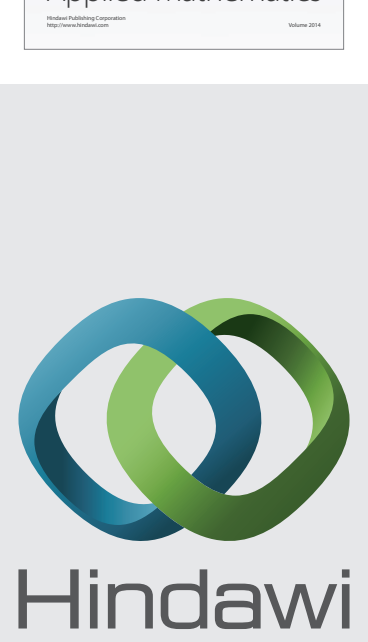

Submit your manuscripts at http://www.hindawi.com
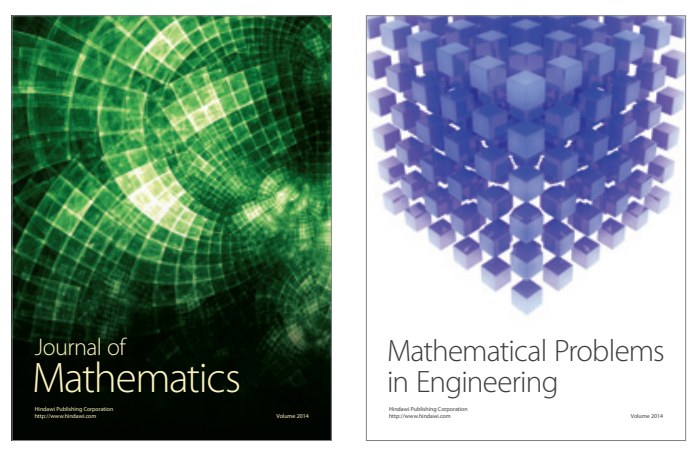

Mathematical Problems in Engineering
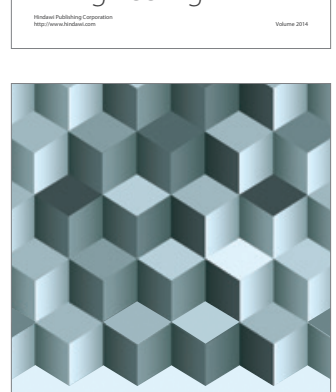

Journal of

Function Spaces
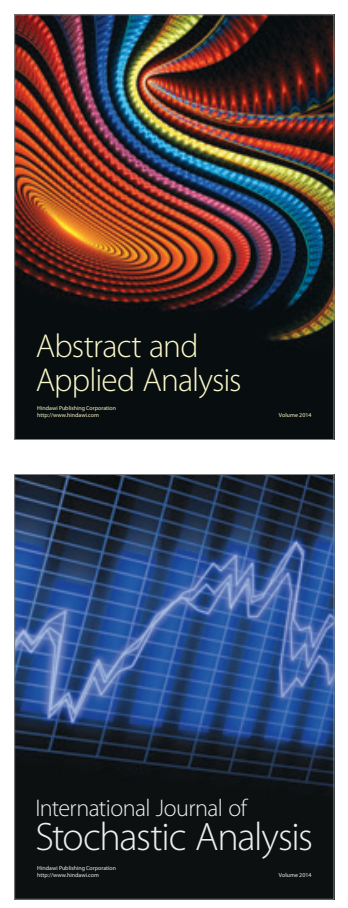

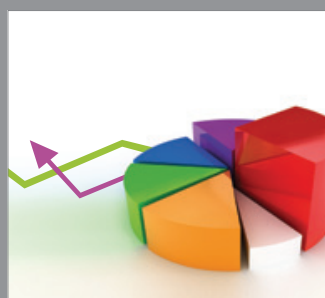

ournal of

Probability and Statistics

Promensencen
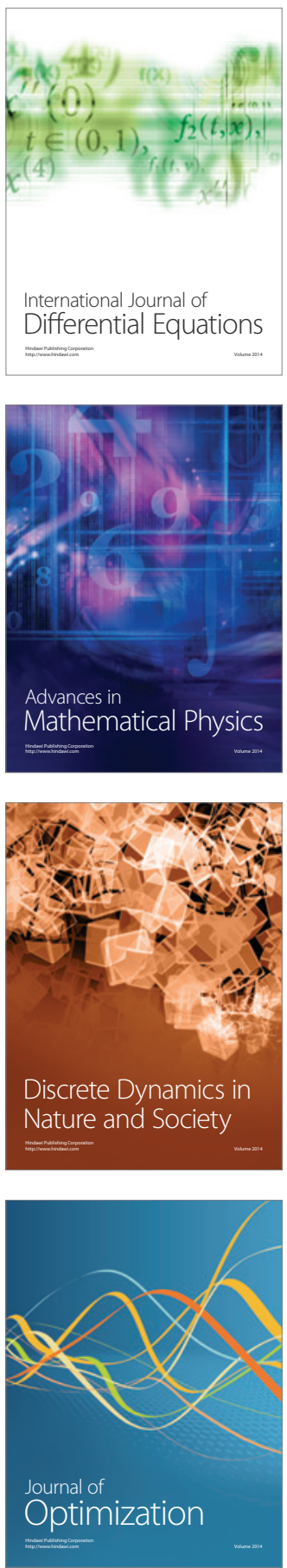